\title{
A Robust Modified Weak Galerkin Finite Element Method for Reaction-Diffusion Equations
}

\author{
Guanrong $\mathrm{Li}^{1}$, Yanping Chen ${ }^{2, *}$ and Yunqing Huang ${ }^{3}$ \\ ${ }^{1}$ School of Mathematics and Statistics, Lingnan Normal University, Zhanjiang \\ 524048, Guangdong, P.R. China \\ 2 School of Mathematical Science, South China Normal University, Guangzhou \\ 510631, Guangdong, P.R. China \\ ${ }^{3}$ Hunan Key Laboratory for Computation and Simulation in Science and \\ Engineering, School of Mathematics and Computational Science, Xiangtan \\ University, Xiangtan 411105, Hunan, P.R. China
}

Received 11 October 2020; Accepted (in revised version) 16 August 2021

\begin{abstract}
In this paper, a robust modified weak Galerkin (MWG) finite element method for reaction-diffusion equations is proposed and investigated. An advantage of this method is that it can deal with the singularly perturbed reaction-diffusion equations. Another advantage of this method is that it produces fewer degrees of freedom than the traditional WG method by eliminating the element boundaries freedom. It is worth pointing out that, in our method, the test functions space is the same as the finite element space, which is helpful for the error analysis. Optimalorder error estimates are established for the corresponding numerical approximation in various norms. Some numerical results are reported to confirm the theory.
\end{abstract}

AMS subject classifications: Primary: 65N15, 65N30; Secondary: 35J50

Key words: Reaction-diffusion equations, singular perturbation, modified weak Galerkin, finite element methods, discrete gradient.

\section{Introduction}

The weak Galerkin (WG) finite element method is a numerical technique for solving partial differential equations. Since it has been proposed by Wang [28], the WG method has been applied successfully to the discretization of several classes of partial differential equations and variational inequalities, e.g., second-order elliptic problems $[2-5,8,9,11,16,19,29,31]$, the Stokes equations [25, 30, 33, 34], the Biharmonic equations [18, 21, 27, 35], the Maxwell equations [22], the Oseen equations [15], the *Corresponding author. Email addresses: yanpingchen@scnu.edu.cn (Y. Chen), liguanrong88@126.com
(G. Li), huangyq@xtu.edu.cn (Y. Huang) 
Helmholtz equations [20,23], the multi-term time-fractional diffusion equations [36], the parabolic integro-differential equations [37], the interface problems [17,24], the natural convection problems [6], and the elliptic variational inequality [26].

It is known that the weak function defined in the WG method has the form $v=$ $\left\{v_{0}, v_{b}\right\}$ with $v=v_{0}$ inside of the element and $v=v_{b}$ on the boundary of the element. The introduction of the weak function and the corresponding weak derivative makes the WG method highly flexible. However, it creates additional degrees of freedom associated $v_{b}$. In order to eliminate the unknowns associated with the element boundaries, Wang [31] and Gao [5] modified the WG method for second-order elliptic problems by replacing $v_{b}$ with the average of $v_{0}:\left\{v_{0}\right\}$. It means that the weak function in the modified weak Galerkin (MWG) method has the form $v=\left\{v_{0},\left\{v_{0}\right\}\right\}$. Therefore, the MWG method has fewer unknowns than the traditional WG method. In [5,31], the finite element space for second-order elliptic problems is defined by

$$
V_{h}:=\left\{v:\left.v\right|_{T} \in P_{k}(T) \text { for } T \in \mathcal{T}_{h}\right\},
$$

and the test function space by

$$
V_{h}^{0}:=\left\{v: v \in V_{h},\left.v\right|_{e}=0 \text { for } e \in \partial \Omega\right\} .
$$

However, the error $e_{h}=u_{h}-Q_{0} u$ between the MWG solution and the $L^{2}$ projection of the exact solution does not necessarily belong to the test function space $V_{h}^{0}$. This will make it difficult to analyse the error.

In this paper, we consider the following diffusion-reaction equations which seeks an unknown function $u=u(\boldsymbol{x})$ satisfying

$$
\begin{array}{cl}
-\nabla \cdot(\mathcal{A} \nabla u)+c u=f & \text { in } \Omega, \\
u=0 & \text { on } \partial \Omega,
\end{array}
$$

where $\Omega$ is a polygonal/plolyhedral domain in $\mathbb{R}^{d}(d=2,3), \mathcal{A}$ is a symmetric matrix, and $c=c(\boldsymbol{x}) \in L^{\infty}(\Omega)$ is a scalar positive function on $\Omega$. Assume that the matrix $\mathcal{A}$ satisfies the following property: there exists a constant $\lambda>0$ such that

$$
\xi^{t} \mathcal{A} \xi \geq \lambda \xi^{t} \xi, \quad \forall \xi \in \mathbb{R}^{d}
$$

where $\xi$ is understood as a column vector and $\xi^{t}$ is the transpose of $\xi$.

Letting $\mathcal{A}=\varepsilon$, where $\varepsilon$ is a constant coefficient and $0<\varepsilon \ll 1$, one can rewrite the problem (1.2)-(1.3) by the following singularly perturbed reaction-diffusion equations: seek an unknown function $u=u(\boldsymbol{x})$ such that

$$
\begin{array}{ll}
-\varepsilon \Delta u+c u=f & \text { in } \Omega, \\
u=0 & \text { on } \partial \Omega .
\end{array}
$$

It is well-known that standard finite element methods often suffer from the deterioration of numerical accuracy for convection-dominated problems. A lot of research has 
been devoted to solving such kinds of problems properly, such as equilibrated residual method [1], anisotropic tetrahedral meshes method [7], discontinuous finite element methods [10], discontinuous Galerkin least-squares finite element methods [12,13].

The goal of this paper is to present a new modified weak Galerkin (MWG) finite method for (1.2)-(1.3). In this MWG method, a difference from the method in [5,31] is that the test functions space is the same as the finite element space. That is, $V_{h}$ defined by (1.1) is not only the finite element space but also the test functions space. This is helpful for the error analysis since the error $e_{h}=u_{h}-Q_{0} u$ belongs to $V_{h}$. It is worth pointing out that the MWG method is accurate and stable even when the solved equations are the singularly perturbed reaction-diffusion equations (1.5)-(1.6).

The rest of the paper is organized as follows. In Section 2, we introduce some preliminaries and notations for Sobolev spaces. In Section 3, we establish a new MWG finite element scheme for the Eqs. (1.2)-(1.3). In Section 4, we give the error equation. In Section 5, we present some technical estimates which are useful for the error analysis. In Section 6, we derive optimal-order error estimates for MWG approximation in both $H^{1}$ and $L^{2}$ norms. In Section 7, we present some numerical results which confirm the theory developed in earlier sections. Finally, conclusions are drawn in Section 8.

\section{Preliminaries and notations}

Let $K$ be any domain in $\mathbb{R}^{d}, d=2,3$. We use the standard definition for the Sobolev space $H^{s}(K)$ and their associated inner products $(\cdot, \cdot)_{s, K}$, norms $\|\cdot\|_{s, K}$, and seminorms $|\cdot|_{s, K}$ for any $s \geq 0$. For instance, for any integer $s \geq 0$, the seminorm $|\cdot|_{s, K}$ is defined by

$$
|v|_{s, K}=\left(\sum_{|\alpha|=s} \int_{K}\left|\partial^{\alpha} v\right|^{2} d K\right)^{\frac{1}{2}}
$$

with the usual notation

$$
\alpha=\left(\alpha_{1}, \cdots, \alpha_{d}\right), \quad|\alpha|=\alpha_{1}+\cdots+\alpha_{d}, \quad \partial^{\alpha}=\prod_{j=1}^{d} \partial_{x_{j}}^{\alpha_{j}} .
$$

The Sobolev norm $\|\cdot\|_{m, K}$ is given by

$$
\|v\|_{m, K}=\left(\sum_{j=0}^{m}|v|_{j, K}^{2}\right)^{\frac{1}{2}}
$$

The space $H^{0}(K)$ coincides with $L^{2}(K)$, for which norm and inner product are denoted by $\|\cdot\|_{K}$ and $(\cdot, \cdot)_{K}$, respectively. If $K=\Omega$, we shall drop the subscript $K$ in the $L^{2}$ norm and the $L^{2}$ inner product notations.

The space $H(\operatorname{div} ; \mathrm{K})$ is given by the set of vector-valued functions on $K$ which, together with their divergence, are square integrable, i.e.,

$$
H(\operatorname{div} ; \mathrm{K}):=\left\{\boldsymbol{v}: \boldsymbol{v} \in\left[\mathrm{L}^{2}(\mathrm{~K})\right]^{\mathrm{d}}, \nabla \cdot \boldsymbol{v} \in \mathrm{L}^{2}(\mathrm{~K})\right\} .
$$


The norm in $H($ div; $\mathrm{K})$ is defined as

$$
\|\boldsymbol{v}\|_{H(\mathrm{div} ; \mathrm{K})}=\left(\|\boldsymbol{v}\|_{K}^{2}+\|\nabla \cdot \boldsymbol{v}\|_{K}^{2}\right)^{\frac{1}{2}}
$$

\section{Modified weak Galerkin finite element schemes}

Let $\mathcal{T}_{h}$ be a partition of the domain $\Omega$ with mesh $h$ that consists of arbitrary polygons/polyhedra. Assume that the partition $\mathcal{T}_{h}$ is WG shape regular defined by a set of conditions as detailed in [14,29]. Denote by $\mathcal{E}_{h}$ the set of all edges/faces in $\mathcal{T}_{h}$, and $\mathcal{E}_{h}^{0}=\mathcal{E}_{h} / \partial \Omega$ the set of all interior edges/faces in $\mathcal{T}_{h}$.

In the weak Galerkin finite element method, the finite dimensional space is defined as

$$
\mathcal{V}_{h}:=\left\{v=\left\{v_{0}, v_{b}\right\}:\left.v_{0}\right|_{T} \in P_{k}(T),\left.v_{b}\right|_{e} \in P_{k}(e), e \subset \partial T, T \in \mathcal{T}_{h}\right\},
$$

where $k \geq 1$. We would like to emphasize that any function $v \in \mathcal{V}_{h}$ has a single value $v_{b}$ on each edge $e \in \mathcal{E}_{h}$. Denote by $\mathcal{V}_{h}^{0}$ the subspace of $\mathcal{V}_{h}$ consisting of discrete weak function with vanishing boundary value, i.e.,

$$
\mathcal{V}_{h}^{0}:=\left\{v=\left\{v_{0}, v_{b}\right\} \in \mathcal{V}_{h}, v_{b}=0 \text { on } \partial \Omega\right\} .
$$

The modified weak Galerkin method will eliminate $v_{b}$ from $\mathcal{V}_{h}$. Thus the finite element space in the MWG formulation is defined as

$$
V_{h}:=\left\{v \in L^{2}(\Omega):\left.v\right|_{T} \in P_{k}(T), T \in \mathcal{T}_{h}\right\},
$$

where $k \geq 1$. We also defined a vector valued polynomial space $W_{h}$ as

$$
W_{h}:=\left\{\boldsymbol{q} \in\left[L^{2}(\Omega)\right]^{2}:\left.\boldsymbol{q}\right|_{T} \in\left[P_{k-1}(T)\right]^{2}, \forall T \in \mathcal{T}_{h}\right\}
$$

where $k \geq 1$.

In order to build a bridge between $\mathcal{V}_{h}$ and $V_{h}$, we need to introduce the average and the jump of the function $v$. For any interior edge/faces $e \in \mathcal{E}_{h}^{0}$, let $T_{1}, T_{2}$ be two elements sharing $e$ and $n_{\mathbf{1}}$ and $\boldsymbol{n}_{\mathbf{2}}$ be the unit outward normal vectors on $e$, associated with $T_{1}$ and $T_{2}$, respectively. For scalar-function $v$ and vector-function $\boldsymbol{q}$, we define their average $\{\cdot\}$ and jump $\llbracket \cdot \rrbracket$ on $e \in \mathcal{E}_{h}$ by

$$
\begin{aligned}
& \{v\}=\left\{\begin{array}{ll}
\frac{1}{2}\left(\left.v\right|_{T_{1}}+\left.v\right|_{T_{2}}\right), & e \in \mathcal{E}_{h}^{0}, \\
0, & e \in \partial \Omega,
\end{array} \quad \llbracket v \rrbracket= \begin{cases}\left.v\right|_{T_{1}} \boldsymbol{n}_{\mathbf{1}}+\left.v\right|_{T_{2}} \boldsymbol{n}_{\mathbf{2}}, & e \in \mathcal{E}_{h}^{0}, \\
v \boldsymbol{n}, & e \in \partial \Omega,\end{cases} \right. \\
& \{\boldsymbol{q}\}=\left\{\begin{array}{ll}
\frac{1}{2}\left(\left.\boldsymbol{q}\right|_{T_{1}}+\left.\boldsymbol{q}\right|_{T_{2}}\right), & e \in \mathcal{E}_{h}^{0}, \\
0, & e \in \partial \Omega,
\end{array} \quad \llbracket \boldsymbol{q} \rrbracket= \begin{cases}\left.\boldsymbol{q}\right|_{T_{1}} \cdot \boldsymbol{n}_{\mathbf{1}}+\left.\boldsymbol{q}\right|_{T_{2}} \cdot \boldsymbol{n}_{\mathbf{2}}, & e \in \mathcal{E}_{h}^{0}, \\
\boldsymbol{q} \cdot \boldsymbol{n}, & e \in \partial \Omega .\end{cases} \right.
\end{aligned}
$$

Thus, $V_{h}$ can be viewed as a subspace of $\mathcal{V}_{h}^{0}$ by the embedding operator: $v \rightarrow\{v,\{v\}\}$. 
Lemma 3.1. Let $\phi, w$ be a scalar-function and a vector-function, respectively. If they are regular enough to make all involving terms well-defined, then,

$$
\sum_{T \in \mathcal{T}_{h}}\langle\boldsymbol{w} \cdot \boldsymbol{n}, \phi-\{\phi\}\rangle_{\partial T}=\sum_{e \in \mathcal{E}_{h}^{0}}\langle\{\boldsymbol{w}\}, \llbracket \phi \rrbracket\rangle_{e}+\sum_{e \in \partial \Omega}\langle\boldsymbol{w} \cdot \boldsymbol{n}, \phi\rangle_{e} .
$$

Proof. For $e \in \mathcal{E}_{h}^{0}$, let $T_{1}$ and $T_{2}$ be two elements sharing $e$ in common. Applying the definition of $\{\cdot\}$ and $\llbracket \cdot \rrbracket$, we have

$$
\begin{aligned}
&\left\langle\left.\boldsymbol{w}\right|_{T_{1}} \cdot \boldsymbol{n}_{\mathbf{1}},\left.\phi\right|_{T_{1}}\right\rangle_{\partial T_{1}}+\left\langle\left.\boldsymbol{w}\right|_{T_{2}} \cdot \boldsymbol{n}_{\mathbf{2}},\left.\phi\right|_{T_{2}}\right\rangle_{\partial T_{2}} \\
&=\left\langle\left.\boldsymbol{w}\right|_{T_{1}} \cdot \boldsymbol{n}_{\mathbf{1}}+\left.\boldsymbol{w}\right|_{T_{2}} \cdot \boldsymbol{n}_{\mathbf{2}},\left.\frac{1}{2} \phi\right|_{T_{1}}\right\rangle_{\partial T_{1}}+\left\langle\left.\boldsymbol{w}\right|_{T_{1}} \cdot \boldsymbol{n}_{\mathbf{1}}+\left.\boldsymbol{w}\right|_{T_{2}} \cdot \mathbf{n}_{\mathbf{2}},\left.\frac{1}{2} \phi\right|_{T_{2}}\right\rangle_{\partial T_{2}} \\
&+\left\langle\left.\boldsymbol{w}\right|_{T_{1}} \cdot \boldsymbol{n}_{\mathbf{1}},\left.\frac{1}{2} \phi\right|_{T_{1}}\right\rangle_{\partial T_{1}}+\left\langle\left.\boldsymbol{w}\right|_{T_{2}} \cdot \boldsymbol{n}_{\mathbf{2}},\left.\frac{1}{2} \phi\right|_{T_{2}}\right\rangle_{\partial T_{2}} \\
&-\left\langle\left.\boldsymbol{w}\right|_{T_{2}} \cdot \boldsymbol{n}_{\mathbf{2}},\left.\frac{1}{2} \phi\right|_{T_{1}}\right\rangle_{\partial T_{1}}-\left\langle\left.\boldsymbol{w}\right|_{T_{1}} \cdot \boldsymbol{n}_{\mathbf{1}},\left.\frac{1}{2} \phi\right|_{T_{2}}\right\rangle_{\partial T_{2}} \\
&=\langle\llbracket \boldsymbol{w} \rrbracket,\{\phi\}\rangle_{e}+\left\langle\left.\frac{1}{2} \boldsymbol{w}\right|_{T_{1}}+\left.\frac{1}{2} \boldsymbol{w}\right|_{T_{2}},\left.\phi\right|_{T_{1}} \mathbf{n}_{\mathbf{1}}\right\rangle_{\partial T_{1}}+\left\langle\left.\frac{1}{2} \boldsymbol{w}\right|_{T_{1}}+\left.\frac{1}{2} \boldsymbol{w}\right|_{T_{2}},\left.\phi\right|_{T_{1}} \boldsymbol{n}_{\mathbf{2}}\right\rangle_{\partial T_{2}} \\
&=\langle\llbracket \boldsymbol{w} \rrbracket,\{\phi\}\rangle_{e}+\langle\{\boldsymbol{w}\}, \llbracket \phi \rrbracket\rangle_{e} .
\end{aligned}
$$

Thus,

$$
\left\langle\left.\boldsymbol{w}\right|_{T_{1}} \cdot \boldsymbol{n}_{\mathbf{1}},\left.\phi\right|_{T_{1}}\right\rangle_{\partial T_{1}}+\left\langle\left.\boldsymbol{w}\right|_{T_{2}} \cdot \boldsymbol{n}_{\mathbf{2}},,\left.\phi\right|_{T_{2}}\right\rangle_{\partial T_{2}}-\langle\llbracket \boldsymbol{w} \rrbracket,\{\phi\}\rangle_{e}=\langle\{\boldsymbol{w}\}, \llbracket \phi \rrbracket\rangle_{e},
$$

which yields the identity (3.1).

The discrete weak gradient operator on $\mathcal{V}_{h}$ is defined in the following sense.

Definition 3.1 ([28]). For each $v=\left\{v_{0}, v_{b}\right\} \in \mathcal{V}_{h}$, the discrete weak gradient operator of $v$, denoted by $\widetilde{\nabla}_{w}$, is defined as the unique piecewise polynomial $\widetilde{\nabla}_{w} v \in W_{h}$ satisfying the following equation:

$$
\left(\widetilde{\nabla}_{w} v, \boldsymbol{q}\right)_{T}=-\left(v_{0}, \nabla \cdot \boldsymbol{q}\right)_{T}+\left\langle v_{b}, \boldsymbol{q} \cdot \boldsymbol{n}\right\rangle_{\partial T}, \quad \forall \boldsymbol{q} \in W_{h},
$$

where $\boldsymbol{n}$ is the unit outward normal to $\partial T$.

By applying the usual integration by part to the first term on the right hand side of (3.2), we can rewrite the Eq. (3.2) as

$$
\left(\widetilde{\nabla}_{w} v, \boldsymbol{q}\right)_{T}=\left(\nabla v_{0}, \boldsymbol{q}\right)_{T}-\left\langle v_{0}-v_{b}, \boldsymbol{q} \cdot \boldsymbol{n}\right\rangle_{\partial T}, \quad \forall \boldsymbol{q} \in W_{h} .
$$

We give the definition of the discrete weak gradient operator on $V_{h}$ in the following sense. 
Definition 3.2. For each $v \in V_{h}$, the discrete weak gradient operator of $v$, denoted by $\nabla_{w}$, is defined as the unique piecewise polynomial $\nabla_{w} v \in W_{h}$ satisfying the following equation:

$$
\left(\nabla_{w} v, \boldsymbol{q}\right)_{T}=-(v, \nabla \cdot \boldsymbol{q})_{T}+\langle\{v\}, \boldsymbol{q} \cdot \boldsymbol{n}\rangle_{\partial T}, \quad \forall \boldsymbol{q} \in W_{h},
$$

where $\boldsymbol{n}$ is the unit outward normal to $\partial T$.

By applying the usual integration by part to the first term on the right hand side of (3.4), we can rewrite the Eq. (3.4) as

$$
\left(\nabla_{w} v, \boldsymbol{q}\right)_{K}=(\nabla v, \boldsymbol{q})_{T}-\langle v-\{v\}, \boldsymbol{q} \cdot \boldsymbol{n}\rangle_{\partial T}, \quad \forall \boldsymbol{q} \in W_{h} .
$$

Let $Q_{0}$ be the local $L^{2}$ projection on $\mathcal{T}_{h}$ and $Q_{b}$ be the local $L^{2}$ projection on $\mathcal{E}_{h}$. Thus, $\left.Q_{0}\right|_{T}$ is the $L^{2}$ projection from $L^{2}(T)$ onto $P_{k}(T)$ and $\left.Q_{b}\right|_{e}$ is the $L^{2}$ projection from $L^{2}(e)$ onto $P_{k}(e)$. For any $v \in H^{1}(\Omega)$, we define the projection operator $Q_{h}$ : $H^{1}(\Omega) \rightarrow \mathcal{V}_{h}$

$$
Q_{h} v=\left\{Q_{0} v, Q_{b} v\right\}
$$

In addition, denote by $\mathbb{Q}_{h}$ the local $L^{2}$ projection onto $\left[P_{k-1}(T)\right]^{d}$. The following lemma presents a useful property which indicates the discrete weak gradient operator is good approximation to the gradient operator in the classical sense.

Lemma 3.2 ([28]). The $L^{2}$-projection $Q_{h}$ and $\mathbb{Q}_{h}$ have the following commutative property:

$$
\widetilde{\nabla}_{w}\left(Q_{h} \phi\right)=\mathbb{Q}_{h}(\nabla \phi), \quad \forall \phi \in H^{1}(T) .
$$

Now we introduce two forms on $V_{h}$ as follows:

$$
\begin{aligned}
& a(v, w)=\left(\mathcal{A} \nabla_{w} v, \nabla_{w} w\right)+(v, w), \\
& s(v, w)=\rho \sum_{e \in \mathcal{E}_{h}} h^{-1}\langle\llbracket v \rrbracket, \llbracket w \rrbracket\rangle_{e},
\end{aligned}
$$

where

$$
\left(\mathcal{A} \nabla_{w} v, \nabla_{w} w\right)=\sum_{T \in \mathcal{T}_{h}}\left(\mathcal{A} \nabla_{w} v, \nabla_{w} w\right)_{T}, \quad(v, w)=\sum_{T \in \mathcal{T}_{h}}(v, w)_{T},
$$

$\rho$ can be any positive number. In practical computation, one might set $\rho=1$. Denote by $a_{s}(\cdot, \cdot)$ a stabilization of $a(\cdot, \cdot)$ given by

$$
a_{s}(v, w)=a(v, w)+s(v, w) .
$$

\section{Algorithm 3.1}

A numerical approximation for the equations (1.2)-(1.3) can be obtained by seeking $u_{h} \in V_{h}$ satisfying the following equation:

$$
a_{s}\left(u_{h}, v\right)=(f, v), \quad \forall v \in V_{h} .
$$


We can see from (3.8) that the MWG and the IPDG [32] have the same type of discrete spaces. However, comparing with IPDG method, there is no need to choose the penalty factor in the MWG method. By (1.4) and the symmetry of $\mathcal{A}$, it is easy to get that the system (3.8) is positive semi-definite for any parameter value of $\rho>0$. Next, we justify the well-postedness of the scheme (3.8). For any $v \in V_{h}$, let

$$
\|v\|:=\sqrt{a_{s}(v, v)}
$$

It is not hard to see that $\|\cdot\|$ defines a semi-norm in the finite element space $V_{h}$. Moreover, we have the following lemma.

Lemma 3.3. $\|\cdot\| \|$ defines a norm on $V_{h}$.

Proof. It suffices to check the positivity property for $\|\cdot\| \cdot \|$. To this end, we need check that for $v \in V_{h}, v=0$ if $\|v\|=0$. In fact, $\|v\|=0$ implies that $\nabla_{w} v=0$ on each element $T$ and $\llbracket v \rrbracket=0$ on each edge $e \in \mathcal{E}_{h}$. From $\llbracket v \rrbracket=0$, it is easy to obtain that $v$ is continuous on $\Omega$ and $v=0$ on $\partial \Omega$. It follows from $\nabla_{w} v=0$ and the Eqs. (3.5) and (3.1) that for any $\boldsymbol{q} \in\left[P_{k-1}(T)\right]^{d}$,

$$
\begin{aligned}
0 & =\sum_{T \in \mathcal{T}_{h}}\left(\nabla_{w} v, \boldsymbol{q}\right)_{T}=\sum_{T \in \mathcal{T}_{h}}(\nabla v, \boldsymbol{q})_{T}-\sum_{T \in \mathcal{T}_{h}}\langle v-\{v\}, \boldsymbol{q} \cdot \boldsymbol{n}\rangle_{\partial T} \\
& =\sum_{T \in \mathcal{T}_{h}}(\nabla v, \boldsymbol{q})_{T}-\sum_{T \in \mathcal{E}_{h}^{0}}\langle\llbracket v \rrbracket,\{\boldsymbol{q}\}\rangle_{e}-\sum_{e \in \partial \Omega}\langle\llbracket v \rrbracket, \boldsymbol{q}\rangle_{e}=(\nabla v, \boldsymbol{q})_{T} .
\end{aligned}
$$

Letting $\boldsymbol{q}=\nabla v$ in the equation above yields $\nabla v=0$ on $T \in \mathcal{T}_{h}$. Thus, $v$ is a constant on each element $T$. This, together with the fact that $v$ is continuous on $\Omega$ and $v=0$ on $\partial \Omega$, implies that $v=0$ on $\Omega$.

Lemma 3.4. The weak Galerkin finite element scheme (3.8) has a unique solution.

Proof. Let $u_{h}^{(1)}$ and $u_{h}^{(2)}$ be two solutions of (3.8). It is clear that the difference $w_{h}=u_{h}^{(1)}-u_{h}^{(2)}$ is a finite element function in $V_{h}$ satisfying

$$
a_{s}\left(w_{h}, v\right)=0, \quad \forall v \in V_{h} .
$$

By setting $v=w_{h}$ in (3.10) we obtain

$$
\left\|w_{h}\right\|^{2}=a_{s}\left(w_{h}, w_{h}\right)=0 .
$$

It follows that $w_{h} \equiv 0$, or equivalently $u_{h}^{(1)}=u_{h}^{(2)}$, which completes the proof. 


\section{Error equations}

The goal of this section is to establish the error equation which can be used to derive the following error estimates for the MWG finite element solution. Let $u_{h} \in V_{h}$ be the MWG finite element solution arising from the numerical scheme (3.8). Assume that the exact solution of the Eqs. (1.2)-(1.3) is given by $u$. Let

$$
e_{h}=u_{h}-Q_{0} u
$$

be the error between the MWG finite element solution and the $L^{2}$ projection of the exact solution.

Lemma 4.1. Let $e_{h}$ be the error defined by (4.1). Then, for any $v \in V_{h}$, one has

$$
\begin{aligned}
a_{s}\left(e_{h}, v\right)= & \left(\mathcal{A} \widetilde{\nabla}_{w}\left(Q_{h} u\right)-\mathcal{A} \nabla_{w}\left(Q_{0} u\right), \nabla_{w} v\right)+c\left(u-Q_{0} u, v\right) \\
& -\sum_{T \in \mathcal{T}_{h}}\left\langle\left(\mathcal{A} \nabla u-\mathcal{A} \mathbb{Q}_{h}(\nabla u)\right) \cdot \boldsymbol{n}, v-\{v\}\right\rangle_{\partial T}-s\left(Q_{0} u, v\right) .
\end{aligned}
$$

Proof. Testing (1.2) by using $v \in V_{h}$, we arrive at

$$
(f, v)=(\mathcal{A} \nabla u, \nabla v)+c(u, v)-\sum_{T \in \mathcal{T}_{h}}\langle(\mathcal{A} \nabla u) \cdot \boldsymbol{n}, v-\{v\}\rangle_{\partial T},
$$

where we have used the fact that

$$
\sum_{T \in \mathcal{T}_{h}}\langle(\mathcal{A} \nabla u) \cdot \boldsymbol{n},\{v\}\rangle_{\partial T}=0 .
$$

For $w \in \mathcal{V}_{h}$, it follows from (3.3) and (3.6) that

$$
\begin{aligned}
& \left(\mathcal{A} \widetilde{\nabla}_{w}\left(Q_{h} u\right), \widetilde{\nabla}_{w} w\right)=\left(\mathcal{A} \mathbb{Q}_{h}(\nabla u), \widetilde{\nabla}_{w} w\right) \\
= & \left.\left(\nabla w_{0}, \mathcal{A} \mathbb{Q}_{h}(\nabla u)\right)-\sum_{T \in \mathcal{T}_{h}}\left\langle w_{0}-w_{b},\left(\mathcal{A} \mathbb{Q}_{h}(\nabla u)\right) \cdot \boldsymbol{n}\right)\right\rangle_{\partial T} \\
= & \left(\mathcal{A} \nabla u, \nabla w_{0}\right)-\sum_{T \in \mathcal{T}_{h}}\left\langle\left(\mathcal{A} \mathbb{Q}_{h}(\nabla u)\right) \cdot \boldsymbol{n}, w_{0}-w_{b}\right\rangle_{\partial T} .
\end{aligned}
$$

Notice that for any $v \in V_{h}$, we have $\{v,\{v\}\} \in \mathcal{V}_{h}$. Thus, we obtain

$$
(\mathcal{A} \nabla u, \nabla v)=\left(\mathcal{A} \widetilde{\nabla}_{w}\left(Q_{h} u\right), \nabla_{w} v\right)+\sum_{T \in \mathcal{T}_{h}}\left\langle\left(\mathcal{A} \mathbb{Q}_{h}(\nabla u)\right) \cdot \boldsymbol{n}, v-\{v\}\right\rangle_{\partial T} .
$$

By (4.3) and (4.4), we obtain

$$
\begin{aligned}
(f, v)= & \left(\mathcal{A} \widetilde{\nabla}_{w}\left(Q_{h} u\right), \nabla_{w} v\right)_{T}+c(u, v) \\
& -\sum_{T \in \mathcal{T}_{h}}\left\langle\left(\mathcal{A} \nabla u-\mathcal{A} \mathbb{Q}_{h}(\nabla u)\right) \cdot \boldsymbol{n}, v-\{v\}\right\rangle_{\partial T} .
\end{aligned}
$$


By the definition of $a(\cdot, \cdot)$, we can get

$$
a\left(Q_{0} u, v\right)=\left(\mathcal{A} \nabla_{w}\left(Q_{0} u\right), \nabla_{w} v\right)+c\left(Q_{0} u, v\right) .
$$

According to (3.8) and the definition of $e_{h}$, we can get

$$
a_{s}\left(e_{h}, v\right)=a_{s}\left(u_{h}, v\right)-a_{s}\left(Q_{0} u, v\right)=(f, v)-a\left(Q_{0} u, v\right)-s\left(Q_{0} u, v\right) .
$$

Combining (4.5)-(4.7), we obtain

$$
\begin{aligned}
a_{s}\left(e_{h}, v\right)= & \left(\mathcal{A} \widetilde{\nabla}_{w}\left(Q_{h} u\right), \nabla_{w} v\right)-\left(\mathcal{A} \nabla_{w}\left(Q_{0} u\right), \nabla_{w} v\right)+c\left(u-Q_{0} u, v\right) \\
& -\sum_{T \in \mathcal{T}_{h}}\left\langle\left(\mathcal{A} \nabla u-\mathcal{A} \mathbb{Q}_{h}(\nabla u)\right) \cdot \boldsymbol{n}, v-\{v\}\right\rangle_{\partial T}-s\left(Q_{0} u, v\right),
\end{aligned}
$$

which completes the proof.

\section{Some technical estimates}

In this section, we shall present some technical results useful for the forthcoming error analysis. To this end, we firstly introduce the trace inequality and inverse inequality on WG shape regular partitions. For more details we refer the reader to [29]. For simplicity of notation, we shall use $\lesssim$ denote less than or equal to up to a constant independent of the mesh size, variables, or other parameters appearing in the inequality.

Lemma 5.1 ([29]). Let $\mathcal{T}_{h}$ be a finite element partition of $\Omega$ that is $W G$ shape regular. Then, there exists a constant $C$ such that for any $T \in \mathcal{T}_{h}$ and edge/face $e \in \partial T$, we have

$$
\|\varphi\|_{e}^{2} \leq C\left(h_{T}^{-1}\|\varphi\|_{T}^{2}+h_{T}\|\nabla \varphi\|_{T}^{2}\right)
$$

where $\varphi \in H^{1}(T)$ is any function.

Lemma 5.2 ([29]). Let $\mathcal{T}_{h}$ be a finite element partition of $\Omega$ that is $W G$ shape regular. Then, there exists a constant $C(n)$ such that

$$
\|\nabla \varphi\|_{T} \leq C(n) h_{T}^{-1}\|\varphi\|_{T}, \quad \forall T \in \mathcal{T}_{h}
$$

for any piecewise polynomial $\varphi$ of degree $n$ on $\mathcal{T}_{h}$.

The following lemma provides estimates for the projection operators $Q_{0}$ and $\mathbb{Q}_{h}$.

Lemma 5.3 ([19,29]). Let $\mathcal{T}_{h}$ be a finite element partition of $\Omega$ that is $W G$ shape regular. Then, for any $\phi \in H^{k+1}(\Omega)$, one has

$$
\sum_{T \in \mathcal{T}_{h}}\left\|\phi-Q_{0} \phi\right\|_{T}^{2} \lesssim h^{2(k+1)}\|\phi\|_{k+1}^{2}
$$




$$
\begin{aligned}
& \sum_{T \in \mathcal{T}_{h}}\left\|\nabla\left(\phi-Q_{0} \phi\right)\right\|_{T}^{2} \lesssim h^{2 k}\|\phi\|_{k+1}^{2}, \\
& \sum_{T \in \mathcal{T}_{h}}\left\|\nabla \phi-\mathbb{Q}_{h}(\nabla \phi)\right\|_{T}^{2} \lesssim h^{2 k}\|\phi\|_{k+1}^{2}, \\
& \sum_{T \in \mathcal{T}_{h}}\left(\left\|\phi-Q_{0} \phi\right\|_{T}^{2}+h_{T}^{2}\left\|\nabla\left(\phi-Q_{0} \phi\right)\right\|_{T}^{2}\right) \lesssim h^{2(k+1)}\|\phi\|_{k+1}^{2} .
\end{aligned}
$$

By using the trace inequality (5.1) and Lemma 5.3, one can obtain the following lemma.

Lemma 5.4 ([9]). Let $\mathcal{T}_{h}$ be a finite element partition of $\Omega$ that is WG shape regular. Then, for any $\phi \in H^{k+1}(\Omega)$, one obtains

$$
\sum_{T \in \mathcal{T}_{h}}\left\|\phi-Q_{0} \phi\right\|_{\partial T}^{2} \lesssim h^{2 k+1}\|\phi\|_{k+1}^{2} .
$$

Lemma 5.5. Let $\mathcal{T}_{h}$ be a finite element partition of $\Omega$ that is $W G$ shape regular. Then, for any $w \in H_{0}^{k+1}(\Omega)$, one obtains

$$
\sum_{T \in \mathcal{T}_{h}}\left\|\widetilde{\nabla}_{w}\left(Q_{h} w\right)-\nabla_{w}\left(Q_{0} w\right)\right\|_{T}^{2} \lesssim h^{2 k}\|w\|_{k+1}^{2} .
$$

Proof. For $\boldsymbol{q} \in W_{h}$ and $T \in \mathcal{T}_{h}$, by (3.2), (3.4) and (5.1)-(5.2), we obtain

$$
\begin{aligned}
& \left|\left(\widetilde{\nabla}_{w}\left(Q_{h} w\right)-\nabla_{w}\left(Q_{0} w\right), \boldsymbol{q}\right)_{T}\right| \\
= & \left|\left\langle Q_{b} w-\left\{Q_{0} w\right\}, \boldsymbol{q} \cdot \boldsymbol{n}\right\rangle_{\partial T}\right| \\
= & \left|\left\langle Q_{b} w-w+\left\{w-Q_{0} w\right\}, \boldsymbol{q} \cdot \boldsymbol{n}\right\rangle_{\partial T}\right| \\
\leq & \left(\left\|Q_{b} w-w\right\|_{\partial T}+\left\|\left\{w-Q_{0} w\right\}\right\|_{\partial T}\right)\|\boldsymbol{q}\|_{\partial T} \\
\lesssim & h^{-\frac{1}{2}}\left(\left\|Q_{0} w-w\right\|_{\partial T}+\left\|\left\{w-Q_{0} w\right\}\right\|_{\partial T}\right)\|\boldsymbol{q}\|_{T} .
\end{aligned}
$$

Letting $\boldsymbol{q}=\widetilde{\nabla}_{w}\left(Q_{h} w\right)-\nabla_{w}\left(Q_{0} w\right)$ in (5.9), we obtain

$$
\left\|\widetilde{\nabla}_{w}\left(Q_{h} w\right)-\nabla_{w}\left(Q_{0} w\right)\right\|_{T} \lesssim h^{-\frac{1}{2}}\left(\left\|Q_{0} w-w\right\|_{\partial T}+\left\|\left\{w-Q_{0} w\right\}\right\|_{\partial T}\right) .
$$

Thus, by (5.7), we have

$$
\begin{aligned}
& \sum_{T \in \mathcal{T}_{h}}\left\|\nabla_{w}\left(Q_{h} w\right)-\nabla_{w}\left(Q_{0} w\right)\right\|_{T}^{2} \\
\lesssim & \sum_{T \in \mathcal{T}_{h}} h^{-1}\left(\left\|Q_{0} w-w\right\|_{\partial T}+\left\|\left\{w-Q_{0} w\right\}\right\|_{\partial T}\right)^{2} \\
\lesssim & \sum_{T \in \mathcal{T}_{h}} h^{-1}\left(\left\|Q_{0} w-w\right\|_{\partial T}\right)^{2} \\
\lesssim & h^{2 k}\|w\|_{k+1}^{2},
\end{aligned}
$$

which completes the proof. 
Lemma 5.6. Let $\mathcal{T}_{h}$ be a finite element partition of $\Omega$ that is $W G$ shape regular. Then, for any $w \in H_{0}^{k+1}(\Omega)$ and $v \in V_{h}$, one has

$$
\begin{aligned}
& \left|h^{-1} \sum_{e \in \mathcal{E}_{h}}\left\langle\llbracket Q_{0} w \rrbracket, \llbracket v \rrbracket\right\rangle\right| \lesssim h^{k}\|v\|\|w\|_{k+1}, \\
& \left|\left(\mathcal{A} \widetilde{\nabla}_{w}\left(Q_{h} w\right)-\mathcal{A} \nabla_{w} Q_{0} w, \nabla_{w} v\right)\right| \lesssim h^{k}\|v\|\|w\|_{k+1}, \\
& \left|\sum_{T \in \mathcal{T}_{h}}\left\langle\left(\mathcal{A} \nabla w-\mathcal{A} \mathbb{Q}_{h}(\nabla w)\right) \cdot \boldsymbol{n}, v-\{v\}\right\rangle_{\partial T}\right| \lesssim h^{k+1}\|v\|\|w\|_{k+1} .
\end{aligned}
$$

Proof. By (5.7) and the fact that $\llbracket w \rrbracket=0$, we can get

$$
\begin{aligned}
&\left|h^{-1} \sum_{e \in \mathcal{E}_{h}}\left\langle\llbracket Q_{0} w \rrbracket, \llbracket v \rrbracket\right\rangle\right|=\left|h^{-1} \sum_{e \in \mathcal{E}_{h}}\left\langle\llbracket Q_{0} w-w \rrbracket, \llbracket v \rrbracket\right\rangle\right| \\
& \leq h^{-1}\left(\sum_{e \in \mathcal{E}_{h}}\left\|\llbracket Q_{0} w-w \rrbracket\right\|_{e}^{2}\right)^{\frac{1}{2}}\left(\sum_{e \in \mathcal{E}_{h}}\|\llbracket v \rrbracket\|_{e}^{2}\right)^{\frac{1}{2}} \\
& \lesssim h^{-1}\left(\sum_{T \in \mathcal{T}_{h}}\left\|Q_{0} w-w\right\|_{\partial T}^{2}\right)^{\frac{1}{2}}\left(\sum_{e \in \mathcal{E}_{h}}\|\llbracket v \rrbracket\|_{e}^{2}\right)^{\frac{1}{2}} \\
&=\left(h^{-1} \sum_{T \in \mathcal{T}_{h}}\left\|Q_{0} w-w\right\|_{\partial T}^{2}\right)^{\frac{1}{2}}\left(\sum_{e \in \mathcal{E}_{h}} h^{-1}\|\llbracket v \rrbracket\|_{e}^{2}\right)^{\frac{1}{2}} \\
& \lesssim h^{k}\|w\|_{k+1}\|v\|,
\end{aligned}
$$

which verifies the inequality (5.10).

By (3.9) and (5.8), one can derive

$$
\begin{aligned}
& \left|\left(\mathcal{A} \widetilde{\nabla}_{w}\left(Q_{h} w\right)-\mathcal{A} \nabla_{w} Q_{0} w, \nabla_{w} v\right)\right| \\
\leq & \left(\sum_{T \in \mathcal{T}_{h}}\left\|\mathcal{A} \nabla_{w}\left(Q_{h} w\right)-\mathcal{A} \nabla_{w} Q_{0} w\right\|^{2}\right)^{\frac{1}{2}}\left(\sum_{T \in \mathcal{T}_{h}}\left\|\nabla_{w} v\right\|^{2}\right)^{\frac{1}{2}} \\
\lesssim & h^{k}\|w\|_{k+1}\|v\|,
\end{aligned}
$$

which completes the proof of the inequality (5.11). By (3.1) and (5.5), we obtain

$$
\begin{aligned}
& \left|\sum_{T \in \mathcal{T}_{h}}\left\langle\left(\mathcal{A} \nabla w-\mathcal{A} \mathbb{Q}_{h}(\nabla w)\right) \cdot \boldsymbol{n}, v-\{v\}\right\rangle_{\partial T}\right| \\
= & \left.\left|\sum_{e \in \mathcal{E}_{h}^{0}}\left\langle\left\{\mathcal{A} \nabla w-\mathcal{A} \mathbb{Q}_{h}(\nabla w)\right\}, \llbracket v \rrbracket\right)\right\rangle_{e}+\sum_{e \in \partial \Omega}\left\langle\mathcal{A} \nabla w-\mathcal{A} \mathbb{Q}_{h}(\nabla w), \llbracket v \rrbracket\right)\right\rangle_{e} \mid \\
\lesssim & \left(\sum_{T \in \mathcal{T}_{h}} h\left\|\mathcal{A} \nabla w-\mathcal{A} \mathbb{Q}_{h}(\nabla w)\right\|_{\partial T}^{2}\right)^{\frac{1}{2}}\left(\sum_{e \in \mathcal{E}_{h}} h^{-1}\|\llbracket v \rrbracket\|_{e}^{2}\right)^{\frac{1}{2}} \lesssim h^{k+1}\|v\|\|w\|_{k+1} .
\end{aligned}
$$


This completes the proof of the inequality (5.12), and hence the lemma.

In the finite element space $V_{h}$, we define the semi-norm $\|\cdot\|_{1, h}$ by

$$
\|v\|_{1, h}=\left(\sum_{T \in \mathcal{T}_{h}}\|\nabla v\|_{T}^{2}+\sum_{e \in \mathcal{E}_{h}} h^{-1}\|\llbracket v \rrbracket\|_{e}^{2}\right)^{\frac{1}{2}}
$$

Lemma 5.7. For any $v \in V_{h}$, we have

$$
\|v\|_{1, h} \lesssim\|v\| .
$$

Proof. For any $v \in V_{h}$, it follows from (3.5) that

$$
\left(\nabla_{w} v, \boldsymbol{q}\right)_{T}=(\nabla v, \boldsymbol{q})_{T}-\langle v-\{v\}, \boldsymbol{q} \cdot \boldsymbol{n}\rangle_{\partial T}, \quad \forall \boldsymbol{q} \in W_{h} .
$$

By setting $\boldsymbol{q}=\nabla v$ in (5.15), we obtain

$$
\left(\nabla_{w} v, \nabla v\right)_{T}=(\nabla v, \nabla v)_{T}-\langle v-\{v\}, \nabla v \cdot \boldsymbol{n}\rangle_{\partial T} .
$$

Thus,

$$
\sum_{T \in \mathcal{T}_{h}}\left(\nabla_{w} v, \nabla v\right)_{T}=\sum_{T \in \mathcal{T}_{h}}(\nabla v, \nabla v)_{T}+\sum_{e \in \mathcal{E}_{h}^{0}}\langle\llbracket v \rrbracket,\{\nabla v\}\rangle_{e}+\sum_{e \in \partial \Omega}\langle\llbracket v \rrbracket, \nabla v\rangle_{e} .
$$

By the trace inequality (5.1) and inverse inequality (5.2), we have

$$
\begin{aligned}
& \sum_{T \in \mathcal{T}_{h}}\|\nabla v\|_{T}^{2} \\
\lesssim & \left(\sum_{T \in \mathcal{T}_{h}}\left\|\nabla_{w} v\right\|_{T}^{2}\right)^{\frac{1}{2}}\left(\sum_{T \in \mathcal{T}_{h}}\|\nabla v\|_{T}^{2}\right)^{\frac{1}{2}}+\left(\sum_{e \in \mathcal{E}_{h}}\|\llbracket v \rrbracket\|_{e}^{2}\right)^{\frac{1}{2}}\left(\sum_{T \in \mathcal{T}_{h}}\|\nabla v\|_{\partial T}^{2}\right)^{\frac{1}{2}} \\
\lesssim & \left(\sum_{T \in \mathcal{T}_{h}}\left\|\nabla_{w} v\right\|_{T}^{2}\right)^{\frac{1}{2}}\left(\sum_{T \in \mathcal{T}_{h}}\|\nabla v\|_{T}^{2}\right)^{\frac{1}{2}}+\left(\sum_{e \in \mathcal{E}_{h}}\|\| v \rrbracket \|_{e}^{2}\right)^{\frac{1}{2}}\left(\sum_{T \in \mathcal{T}_{h}} h^{-1}\|\nabla v\|_{T}^{2}\right)^{\frac{1}{2}} .
\end{aligned}
$$

Thus,

$$
\left(\sum_{T \in \mathcal{T}_{h}}\|\nabla v\|_{T}^{2}\right)^{\frac{1}{2}} \lesssim\left(\sum_{T \in \mathcal{T}_{h}}\left\|\nabla_{w} v\right\|_{T}^{2}\right)^{\frac{1}{2}}+\left(\sum_{e \in \mathcal{E}_{h}} h^{-1}\|\llbracket v \rrbracket\|_{e}^{2}\right)^{\frac{1}{2}} .
$$

This leads to

$$
\sum_{T \in \mathcal{T}_{h}}\|\nabla v\|_{T}^{2} \lesssim \sum_{T \in \mathcal{T}_{h}}\left\|\nabla_{w} v\right\|_{T}^{2}+\sum_{e \in \mathcal{E}_{h}} h^{-1}\|\llbracket v \rrbracket\|_{e}^{2}
$$

Thus,

$$
\|v\|_{1, h}^{2} \lesssim\|v\|^{2}
$$

which completes the proof. 


\section{Error estimates}

The goal of this section is to establish some error estimates for the MWG finite element solution $u_{h}$ arising from (3.8). The error will be measured in the triple-bar norm $\|\cdot\|$ defined by (3.9), the semi-norm $\|\cdot\|_{h}$ defined by (5.13) and the standard $L^{2}$ norm.

Theorem 6.1. Let $u_{h} \in V_{h}$ be the modified weak Galerkin finite element solution of the Eqs. (1.2)-(1.3) arising from (3.8). Assume the exact solution $u \in H^{k+1}(\Omega)$. Then

$$
\left\|u_{h}-Q_{0} u\right\| \lesssim h^{k}\|u\|_{k+1} .
$$

Proof. By letting $v=e_{h}$ in (4.2), we have

$$
\begin{aligned}
\left\|e_{h}\right\|^{2}= & \left(\mathcal{A} \widetilde{\nabla}_{w}\left(Q_{h} u\right)-\mathcal{A} \nabla_{w}\left(Q_{0} u\right), \nabla_{w} e_{h}\right)+c\left(u-Q_{0} u, e_{h}\right) \\
& -\sum_{T \in \mathcal{T}_{h}}\left\langle\left(\mathcal{A} \nabla u-\mathcal{A} \mathbb{Q}_{h}(\nabla u)\right) \cdot \boldsymbol{n}, e_{h}-\left\{e_{h}\right\}\right\rangle_{\partial T}-s\left(Q_{0} u, e_{h}\right) .
\end{aligned}
$$

Applying the definition of $\|\cdot\|$ and the approximation property of the $L^{2}$ - projection, we derive

$$
\begin{aligned}
\left|c\left(u-Q_{0} u, e_{h}\right)\right| & \lesssim\left(\sum_{T \in \mathcal{T}_{h}}\left\|u-Q_{0} u\right\|_{T}^{2}\right)^{\frac{1}{2}}\left(\sum_{T \in \mathcal{T}_{h}}\left\|e_{h}\right\|_{T}^{2}\right)^{\frac{1}{2}} \\
& \lesssim h^{k+1}\|u\|_{k+1}\left\|e_{h}\right\| .
\end{aligned}
$$

Applying (5.10)-(5.12) and (6.3), we have

$$
\left\|e_{h}\right\|^{2} \lesssim h^{k}\|u\|_{k+1}\left\|e_{h}\right\|
$$

which completes the proof.

Next, we will measure the difference between $u$ and $u_{h}$ in the semi-norm $\|\cdot\|_{1, h}$ as defined in (5.13).

Corollary 6.1. Let $u_{h} \in V_{h}$ be the modified weak Galerkin finite element solution of the Eqs. (1.2)-(1.3) arising from (3.8). Assume the exact solution $u \in H^{k+1}(\Omega)$. Then

$$
\left\|u-u_{h}\right\|_{1, h} \lesssim h^{k}\|u\|_{k+1} .
$$

Proof. Applying (5.14) and (6.1), we obtain

$$
\left\|u_{h}-Q_{0} u\right\|_{1, h} \lesssim\left\|u_{h}-Q_{0} u\right\| \lesssim h^{k}\|u\|_{k+1} .
$$

With the definition of discrete $H^{1}$ semi-norm and the estimates (5.4) and (5.7), we derive

$$
\left\|u-Q_{0} u\right\|_{1, h}^{2}=\sum_{T \in \mathcal{T}_{h}}\left\|\nabla\left(u-Q_{0} u\right)\right\|_{T}^{2}+\sum_{e \in \mathcal{E}_{h}} h^{-1}\left\|\llbracket u-Q_{0} u \rrbracket\right\|_{e}^{2}
$$




$$
\begin{aligned}
& \leq \sum_{T \in \mathcal{T}_{h}}\left\|\nabla\left(u-Q_{0} u\right)\right\|_{T}^{2}+\sum_{T \in \mathcal{T}_{h}} h^{-1}\left\|u-Q_{0} u\right\|_{\partial T}^{2} \\
& \lesssim h^{2 k}\|u\|_{k+1}^{2} .
\end{aligned}
$$

Thus,

$$
\left\|u-Q_{0} u\right\|_{1, h} \lesssim h^{k}\|u\|_{k+1} .
$$

It follows from the triangle inequality, and the estimates (6.5) and (6.6) that

$$
\left\|u-u_{h}\right\|_{1, h} \leq\left\|u-Q_{0} u\right\|_{1, h}+\left\|u_{h}-Q_{0} u\right\|_{1, h} \lesssim h^{k}\|u\|_{k+1} .
$$

This completes the proof.

In the rest of the section, we shall establish an optimal-order error for the weak Galerkin finite element scheme (3.8) in the usual $L^{2}$ norm by using a duality argument. To this end, we consider a dual problem that seeks $\psi \in H_{0}^{1}(\Omega) \cap H^{2}(\Omega)$ satisfying

$$
-\nabla \cdot(\mathcal{A} \nabla \psi)+c \psi=e_{h} \quad \text { in } \Omega .
$$

Assume that the above dual problem has the usual $H^{2}$-regularity. This means that

$$
\|\psi\|_{2} \lesssim\left\|e_{h}\right\|
$$

Theorem 6.2. Let $u_{h} \in V_{h}$ be modified weak Galerkin finite element solution of the Eqs. (1.2)-(1.3) arising from (3.8). Assume the exact solution $u \in H^{k+1}(\Omega)$. In addition, assume that the dual problem (6.7) has the usual $H^{2}$-regularity. Then

$$
\left\|u-u_{h}\right\| \lesssim h^{k+1}\|u\|_{k+1}
$$

Proof. By testing (6.7) with $e_{h}$, we obtain

$$
\begin{aligned}
\left\|e_{h}\right\|^{2} & =-\left(\nabla \cdot(\mathcal{A} \nabla \psi), e_{h}\right)+\left(c \psi, e_{h}\right) \\
& =\left(\mathcal{A} \nabla \psi, \nabla e_{h}\right)-\sum_{T \in \mathcal{T}_{h}}\left\langle(\mathcal{A} \nabla \psi) \cdot \boldsymbol{n}, e_{h}-\left\{e_{h}\right\}\right\rangle_{\partial T}+\left(c \psi, e_{h}\right) .
\end{aligned}
$$

Here we have used the fact $\left\langle(\mathcal{A} \nabla \psi) \cdot \boldsymbol{n},\left\{e_{h}\right\}\right\rangle_{\partial T}=0$. Setting $u=\psi$ and $v=e_{h}$ in (4.4), we can obtain the following equation:

$$
\left(\mathcal{A} \nabla \psi, \nabla e_{h}\right)=\left(\mathcal{A} \widetilde{\nabla}_{w}\left(Q_{h} \psi\right), \nabla_{w} e_{h}\right)+\sum_{T \in \mathcal{T}_{h}}\left\langle\left(\mathcal{A} \mathbb{Q}_{h}(\nabla \psi)\right) \cdot \boldsymbol{n}, e_{h}-\left\{e_{h}\right\}\right\rangle_{\partial T}
$$

According the definition of $a_{s}(v, w)$ in (3.7) and the symmetry of $\mathcal{A}$, we can get

$$
a_{s}\left(e_{h}, Q_{0} \psi\right)=\left(\mathcal{A} \nabla_{w}\left(Q_{0} \psi\right), \nabla_{w} e_{h}\right)+\left(c e_{h}, Q_{0} \psi\right)+s\left(e_{h}, Q_{0} \psi\right) .
$$

By combining the (6.10)-(6.12), we can obtain

$$
\left\|e_{h}\right\|^{2}=a_{s}\left(e_{h}, Q_{0} \psi\right)+\left(\mathcal{A} \widetilde{\nabla}_{w}\left(Q_{h} \psi\right), \nabla_{w} e_{h}\right)-\left(\mathcal{A} \nabla_{w}\left(Q_{0} \psi\right), \nabla_{w} e_{h}\right)
$$




$$
\begin{aligned}
& -\sum_{T \in \mathcal{T}_{h}}\left\langle\left(\mathcal{A} \nabla \psi-\mathcal{A} \mathbb{Q}_{h}(\nabla \psi)\right) \cdot \boldsymbol{n}, e_{h}-\left\{e_{h}\right\}\right\rangle_{\partial T} \\
& -s\left(e_{h}, Q_{0} \psi\right)+\left(c e_{h}, \psi-Q_{0} \psi\right) .
\end{aligned}
$$

By (4.2), we derive

$$
\begin{aligned}
\left\|e_{h}\right\|^{2}= & \left(\mathcal{A} \widetilde{\nabla}_{w}\left(Q_{h} u\right)-\mathcal{A} \nabla_{w}\left(Q_{0} u\right), \nabla_{w}\left(Q_{0} \psi\right)\right)+c\left(u-Q_{0} u, Q_{0} \psi\right) \\
& -\sum_{T \in \mathcal{T}_{h}}\left\langle\left(\mathcal{A} \nabla u-\mathcal{A} \mathbb{Q}_{h}(\nabla u)\right) \cdot \boldsymbol{n}, Q_{0} \psi-\left\{Q_{0} \psi\right\}\right\rangle_{\partial T}-s\left(Q_{0} u, Q_{0} \psi\right) \\
& +\left(\mathcal{A} \widetilde{\nabla}_{w}\left(Q_{h} \psi\right)-\mathcal{A} \nabla_{w}\left(Q_{0} \psi\right), \nabla_{w} e_{h}\right)+\left(c e_{h}, \psi-Q_{0} \psi\right) \\
& -\sum_{T \in \mathcal{T}_{h}}\left\langle\left(\mathcal{A} \nabla \psi-\mathcal{A} \mathbb{Q}_{h}(\nabla \psi)\right) \cdot \boldsymbol{n}, e_{h}-\left\{e_{h}\right\}\right\rangle_{\partial T}-s\left(e_{h}, Q_{0} \psi\right) \\
= & \pi_{1}+\pi_{2}+\pi_{3}+\pi_{4}+\pi_{5}+\pi_{6}+\pi_{7}+\pi_{8} .
\end{aligned}
$$

According to Lemma 3.6, we obtain

$$
\begin{aligned}
\left|\pi_{1}\right|= & \left|\left(\mathcal{A} \widetilde{\nabla}_{w}\left(Q_{h} u\right)-\mathcal{A} \nabla_{w}\left(Q_{0} u\right), \nabla_{w}\left(Q_{0} \psi\right)\right)\right| \\
\leq & \left|\left(\mathcal{A} \widetilde{\nabla}_{w}\left(Q_{h} u\right)-\mathcal{A} \nabla_{w}\left(Q_{0} u\right), \nabla_{w}\left(Q_{0} \psi\right)-\widetilde{\nabla}_{w}\left(Q_{h} \psi\right)\right)\right| \\
& +\left|\left(\mathcal{A} \widetilde{\nabla}_{w}\left(Q_{h} u\right)-\mathcal{A} \nabla_{w}\left(Q_{0} u\right), \widetilde{\nabla}_{w}\left(Q_{h} \psi\right)\right)\right| \\
= & \left|\left(\mathcal{A} \widetilde{\nabla}_{w}\left(Q_{h} u\right)-\mathcal{A} \nabla_{w}\left(Q_{0} u\right), \nabla_{w}\left(Q_{0} \psi\right)-\widetilde{\nabla}_{w}\left(Q_{h} \psi\right)\right)\right| \\
& +\left|\left(\mathcal{A} \widetilde{\nabla}_{w}\left(Q_{h} u\right)-\mathcal{A} \nabla_{w}\left(Q_{0} u\right), \mathbb{Q}_{h}(\nabla \psi)\right)\right| \\
= & R_{1}+R_{2} .
\end{aligned}
$$

By (5.8), we have

$$
\begin{aligned}
R_{1} & \lesssim\left(\sum_{T \in \mathcal{T}_{h}}\left\|\mathcal{A} \widetilde{\nabla}_{w}\left(Q_{h} u\right)-\mathcal{A} \nabla_{w}\left(Q_{0} u\right)\right\|_{T}^{2}\right)^{\frac{1}{2}}\left(\sum_{T \in \mathcal{T}_{h}}\left\|\nabla_{w}\left(Q_{0} \psi\right)-\widetilde{\nabla}_{w}\left(Q_{h} \psi\right)\right\|_{T}^{2}\right)^{\frac{1}{2}} \\
& \lesssim h^{k+1}\|u\|_{k+1}\|\psi\|_{2} .
\end{aligned}
$$

By (5.5) and (5.7), we obtain

$$
\begin{aligned}
R_{2} & =\left|\sum_{T \in \mathcal{T}_{h}}\left\langle Q_{b} u-\left\{Q_{0} u\right\},\left(\mathcal{A} \mathbb{Q}_{h}(\nabla \psi)\right) \cdot \boldsymbol{n}\right\rangle_{\partial T}\right| \\
& =\left|\sum_{T \in \mathcal{T}_{h}}\left\langle Q_{b} u-\left\{Q_{0} u\right\},\left(\mathcal{A} \mathbb{Q}_{h}(\nabla \psi)-\mathcal{A} \nabla \psi\right) \cdot \boldsymbol{n}\right\rangle_{\partial T}\right| \\
& \lesssim \sum_{T \in \mathcal{T}_{h}} h^{-\frac{1}{2}}\left\|u-Q_{0} u\right\|_{\partial T}\left\|\mathbb{Q}_{h}(\nabla \psi)-\nabla \psi\right\|_{T}
\end{aligned}
$$




$$
\begin{aligned}
& \lesssim\left(\sum_{T \in \mathcal{T}_{h}} h^{-1}\left\|u-Q_{0} u\right\|_{\partial T}^{2}\right)^{\frac{1}{2}}\left(\sum_{T \in \mathcal{T}_{h}}\left\|\mathbb{Q}_{h}(\nabla \psi)-\nabla \psi\right\|_{T}^{2}\right)^{\frac{1}{2}} \\
& \lesssim h^{k+1}\|u\|_{k+1}\|\psi\|_{2} .
\end{aligned}
$$

Combining (6.14)-(6.15), we have

$$
\left|\pi_{1}\right| \lesssim h^{k+1}\|u\|_{k+1}\|\psi\|_{2} .
$$

By (5.3), we have

$$
\begin{aligned}
\left|\pi_{2}\right|= & \left|c\left(u-Q_{0} u, Q_{0} \psi\right)\right| \\
\lesssim & \sum_{T \in \mathcal{T}_{h}}\left\|u-Q_{0} u\right\|_{T}\left(\left\|Q_{0} \psi-\psi\right\|_{T}+\|\psi\|_{T}\right) \\
= & \sum_{T \in \mathcal{T}_{h}}\left\|u-Q_{0} u\right\|_{T}\left\|Q_{0} \psi-\psi\right\|_{T}+\sum_{T \in \mathcal{T}_{h}}\left\|u-Q_{0} u\right\|_{T}\|\psi\|_{T} \\
\lesssim & \left(\sum_{T \in \mathcal{T}_{h}}\left\|u-Q_{0} u\right\|_{T}^{2}\right)^{\frac{1}{2}}\left(\sum_{T \in \mathcal{T}_{h}}\left\|Q_{0} \psi-\psi\right\|_{T}^{2}\right)^{\frac{1}{2}} \\
& +\left(\sum_{T \in \mathcal{T}_{h}}\left\|u-Q_{0} u\right\|_{T}^{2}\right)^{\frac{1}{2}}\left(\sum_{T \in \mathcal{T}_{h}}\|\psi\|_{T}^{2}\right)^{\frac{1}{2}} \\
& \lesssim h^{k+3}\|u\|_{k+1}\|\psi\|_{2}+h^{k+1}\|u\|_{k+1}\|\psi\|_{2} \\
\leq & h^{k+1}\|u\|_{k+1}\|\psi\|_{2} .
\end{aligned}
$$

By (5.5) and (5.7), we obtain

$$
\begin{aligned}
\left|\pi_{3}\right| & =\left|-\sum_{T \in \mathcal{T}_{h}}\left\langle\left(\mathcal{A} \nabla u-\mathcal{A} \mathbb{Q}_{h}(\nabla u)\right) \cdot \boldsymbol{n}, Q_{0} \psi-\left\{Q_{0} \psi\right\}\right\rangle_{\partial T}\right| \\
& =\left|\sum_{T \in \mathcal{T}_{h}}\left\langle\left(\mathcal{A} \nabla u-\mathcal{A} \mathbb{Q}_{h}(\nabla u)\right) \cdot \boldsymbol{n}, Q_{0} \psi-\psi+\left\{\psi-Q_{0} \psi\right\}\right\rangle_{\partial T}\right| \\
& \left.\leq \sum_{T \in \mathcal{T}_{h}} \| \mathcal{A} \nabla u-\mathcal{A} \mathbb{Q}_{h}(\nabla u)\right)\left\|_{\partial T}\right\| Q_{0} \psi-\psi+\left\{\psi-Q_{0} \psi\right\} \|_{\partial T} \\
& \lesssim\left(\sum_{T \in \mathcal{T}_{h}}\left\|\mathcal{A} \nabla u-\mathcal{A} \mathbb{Q}_{h}(\nabla u)\right\|_{\partial T}^{2}\right)^{\frac{1}{2}}\left(\sum_{T \in \mathcal{T}_{h}}\left\|\psi-Q_{0} \psi\right\|_{\partial T}^{2}\right)^{\frac{1}{2}} \\
& \lesssim h^{k+1}\|u\|_{k+1}\|\psi\|_{2} .
\end{aligned}
$$

By (5.7) and the fact that $\llbracket u \rrbracket=0$ and $\llbracket \psi \rrbracket=0$, we can get

$$
\left|\pi_{4}\right|=\left|s\left(Q_{0} u, Q_{0} \psi\right)\right|=\left|h^{-1} \sum_{e \in \mathcal{E}_{h}}\left\langle\llbracket Q_{0} w-w \rrbracket, \llbracket Q_{0} \psi-\psi \rrbracket\right\rangle\right|
$$




$$
\begin{aligned}
& \leq h^{-1} \sum_{T \in \mathcal{T}_{h}}\left\|Q_{0} w-w\right\|_{\partial T}\left\|Q_{0} \psi-\psi\right\|_{\partial T} \\
& \leq\left(h^{-1} \sum_{T \in \mathcal{T}_{h}}\left\|Q_{0} w-w\right\|_{\partial T}\right)^{\frac{1}{2}}\left(h^{-1} \sum_{T \in \mathcal{T}_{h}}\left\|Q_{0} \psi-\psi\right\|_{\partial T}\right)^{\frac{1}{2}} \\
& \lesssim h^{k+1}\|u\|_{k+1}\|\psi\|_{2} .
\end{aligned}
$$

By (5.10)-(5.12) and (6.1), we obtain the following estimates:

$$
\begin{aligned}
\left|\pi_{5}\right| & =\left|\left(\mathcal{A} \widetilde{\nabla}_{w}\left(Q_{h} \psi\right)-\mathcal{A} \nabla_{w}\left(Q_{0} \psi\right), \nabla_{w} e_{h}\right)\right| \\
& \lesssim h\|\psi\|_{2}\left\|e_{h}\right\| \lesssim h^{k+1}\|u\|_{k+1}\|\psi\|_{2}, \\
\left|\pi_{6}\right| & =\left|\left(c e_{h}, \psi-Q_{0} \psi\right)\right| \lesssim\left(\sum_{T \in \mathcal{T}_{h}}\left\|\psi-Q_{0} \psi\right\|_{T}\right)^{\frac{1}{2}}\left(\sum_{T \in \mathcal{T}_{h}}\left\|e_{h}\right\|_{T}\right)^{\frac{1}{2}} \\
& \leq\left(\sum_{T \in \mathcal{T}_{h}}\left\|\psi-Q_{0} \psi\right\|_{T}\right)^{\frac{1}{2}}\left\|e_{h}\right\| \lesssim h^{k+2}\|u\|_{k+1}\|\psi\|_{2}, \\
\left|\pi_{7}\right| & =\left|-\sum_{T \in \mathcal{T}_{h}}\left\langle\left(\mathcal{A} \nabla \psi-\mathcal{A} \mathbb{Q}_{h}(\nabla \psi)\right) \cdot \boldsymbol{n}, e_{h}-\left\{e_{h}\right\}\right\rangle_{\partial T}\right| \\
& \lesssim h^{2}\|\psi\|_{2}\left\|e_{h}\right\| \lesssim h^{k+2}\|u\|_{k+1}\|\psi\|_{2}, \\
\left|\pi_{8}\right| & =\left|s\left(e_{h}, Q_{0} \psi\right)\right| \lesssim h\|\psi\|_{2}\left\|e_{h}\right\| \lesssim h^{k+1}\|u\|_{k+1}\|\psi\|_{2} .
\end{aligned}
$$

Thus, together with the fact that $\|\psi\|_{2} \leq\left\|e_{h}\right\|$, we obtain

$$
\left\|u-u_{h}\right\| \lesssim h^{k+1}\|u\|_{k+1},
$$

which completes the proof.

\section{Numerical experiments}

The goal of this section is to report some numerical results for the modified weak Galerkin finite element scheme proposed and analyzed in previous sections. For simplicity, we consider a rectangular domain $\Omega=[0,1] \times[0,1]$ with uniform triangulation in this section. The triangular mesh is constructed by: 1) uniformly partitioning the domain into $n \times n$ sub-rectangles; 2) dividing each rectangular element by diagonal line with a negative slope. The mesh size is denoted by $h=\frac{1}{n}$. All of the examples given below will use these triangulations of $\Omega$, and will apply the MWG method to find a numerical solution $u_{h}$ where $\left.u_{h}\right|_{T} \in P_{1}(T)$. Let $u$ be the exact solution to the original equations (1.2)-(1.3). We define the error by $e_{h}=u_{h}-Q_{0} u$ where $Q_{0} u$ is local $L^{2}$ projection on $\mathcal{T}_{h}$. The numerical errors will be measured in the following norms:

$$
\left\|e_{h}\right\|^{2}=\sum_{T \in \mathcal{T}_{h}}\left(\int_{T} \mathcal{A}\left|\nabla_{w} e_{h}\right|^{2} d T+\int_{T} c\left|e_{h}\right|^{2} d T\right)+\sum_{e \in \mathcal{E}_{h}}\left(h^{-1} \int_{e}\left|\llbracket e_{h} \rrbracket\right|^{2} d s\right),
$$




$$
\left\|u-u_{h}\right\|^{2}=\sum_{T \in \mathcal{T}_{h}}\left(\int_{T}\left|u-u_{h}\right|^{2}\right) d T .
$$

Note that $\|\cdot\|$ is a discrete $H^{1}$-norm, and $\|\cdot\|$ is the standard $L^{2}$-norm. The mesh generation and computation are all conducted in the MATLAB environment.

\subsection{Example 1}

In this example, we consider the following singularly perturbed reaction-diffusion equations:

$$
\begin{array}{cl}
-\epsilon \Delta u+u=f & \text { in } \Omega, \\
u=0 & \text { on } \partial \Omega,
\end{array}
$$

where $0<\epsilon \leq 1$. The term $f$ is chosen according the exact solution $u=\sin (\pi x) \sin (\pi y)$. We computed the MWG solutions for $\epsilon=1, \epsilon=10^{-3}$ and $\epsilon=10^{-9}$, respectively. The numerical results are shown in Tables 1 and 2, which indicate that the MWG solutions are convergent with order $\mathcal{O}(h)$ and $\mathcal{O}\left(h^{2}\right)$ in discrete $H^{1}$-norm and $L^{2}$-norm, respectively.

Table 1: Discrete $H^{1}$-norm error and convergence rate for Example 1.

\begin{tabular}{||c|c|c|c|c|c|c||}
\hline$h$ & \multicolumn{2}{|c|}{$\left\|e_{h}\right\|$} & order & $\left\|e_{h}\right\|$ & order & \multicolumn{2}{|c||}{$\left\|e_{h}\right\|$} & order \\
\hline \multicolumn{2}{|c|}{$\epsilon=1$} & \multicolumn{2}{|c|}{$\epsilon=10^{-3}$} & \multicolumn{2}{c||}{$\epsilon=10^{-9}$} \\
\hline$\frac{1}{4}$ & $4.479 \mathrm{e}-01$ & & $3.794 \mathrm{e}-01$ & & $3.797 \mathrm{e}-01$ & \\
\hline$\frac{1}{8}$ & $2.046 \mathrm{e}-01$ & 1.13 & $1.567 \mathrm{e}-01$ & 1.28 & $1.570 \mathrm{e}-01$ & 1.27 \\
\hline$\frac{1}{16}$ & $9.690 \mathrm{e}-02$ & 1.08 & $6.853 \mathrm{e}-02$ & 1.19 & $6.873 \mathrm{e}-02$ & 1.19 \\
\hline$\frac{1}{32}$ & $4.671 \mathrm{e}-02$ & 1.05 & $3.136 \mathrm{e}-02$ & 1.13 & $3.148 \mathrm{e}-02$ & 1.13 \\
\hline$\frac{1}{64}$ & $2.280 \mathrm{e}-02$ & 1.03 & $1.487 \mathrm{e}-02$ & 1.08 & $1.494 \mathrm{e}-02$ & 1.08 \\
\hline$\frac{1}{128}$ & $1.128 \mathrm{e}-02$ & 1.01 & $7.223 \mathrm{e}-03$ & 1.04 & $7.259 \mathrm{e}-03$ & 1.04 \\
\hline
\end{tabular}

Table 2: $L^{2}$-norm error and convergence rate for Example 1 .

\begin{tabular}{||c|c|c|c|c|c|c|c||}
\hline$h$ & $\left\|u_{h}-u\right\|$ & order & $\left\|u_{h}-u\right\|$ & order & $\left\|u_{h}-u\right\|$ & order \\
\hline \multicolumn{2}{|c|}{$\epsilon=1$} & \multicolumn{2}{|c|}{$\epsilon=10^{-3}$} & \multicolumn{2}{c||}{$\epsilon=10^{-9}$} \\
\hline$\frac{1}{4}$ & $5.556 \mathrm{e}-02$ & & $3.509 \mathrm{e}-02$ & & $3.503 \mathrm{e}-02$ & \\
\hline$\frac{1}{8}$ & $1.115 \mathrm{e}-02$ & 2.32 & $7.539 \mathrm{e}-03$ & 2.22 & $7.482 \mathrm{e}-03$ & 2.23 \\
\hline$\frac{1}{16}$ & $2.277 \mathrm{e}-03$ & 2.29 & $1.754 \mathrm{e}-03$ & 2.10 & $1.730 \mathrm{e}-03$ & 2.11 \\
\hline$\frac{1}{32}$ & $4.994 \mathrm{e}-04$ & 2.19 & $4.312 \mathrm{e}-04$ & 2.02 & $4.166 \mathrm{e}-04$ & 2.05 \\
\hline$\frac{1}{64}$ & $1.163 \mathrm{e}-04$ & 2.10 & $1.071 \mathrm{e}-04$ & 2.01 & $1.022 \mathrm{e}-04$ & 2.03 \\
\hline$\frac{1}{128}$ & $2.802 \mathrm{e}-05$ & 2.05 & $2.672 \mathrm{e}-05$ & 2.00 & $2.533 \mathrm{e}-05$ & 2.01 \\
\hline
\end{tabular}




\subsection{Example 2}

In this example, we let the exact solution to the Eqs. (7.1)-(7.2) be

$$
u=x y(1-x)(1-y) \exp (x-y) .
$$

The right-hand side $f$ is chosen according to the exact solution (7.3). We computed the MWG solutions for $\epsilon=1, \epsilon=10^{-3}$ and $\epsilon=10^{-9}$, respectively. The numerical results are shown in Tables 3 and 4, which indicate that the MWG solutions are convergent with order $\mathcal{O}(h)$ and $\mathcal{O}\left(h^{2}\right)$ in discrete $H^{1}$-norm and $L^{2}$-norm, respectively.

Table 3: Discrete $H^{1}$-norm error and convergence rate for Example 2.

\begin{tabular}{||c|c|c|c|c|c|c||}
\hline$h$ & \multicolumn{2}{|c|}{$\left\|e_{h}\right\|$} & order & $\left\|e_{h}\right\|$ & order & \multicolumn{2}{|c||}{$\left\|e_{h}\right\|$} & order \\
\hline \multicolumn{2}{|c|}{$\epsilon=1$} & \multicolumn{2}{|c|}{$\epsilon=10^{-3}$} & \multicolumn{2}{c||}{$\epsilon=10^{-9}$} \\
\hline$\frac{1}{4}$ & $4.891 \mathrm{e}-02$ & & $4.528 \mathrm{e}-02$ & & $4.529 \mathrm{e}-02$ & \\
\hline$\frac{1}{8}$ & $2.195 \mathrm{e}-02$ & 1.16 & $1.916 \mathrm{e}-02$ & 1.24 & $1.918 \mathrm{e}-02$ & 1.24 \\
\hline$\frac{1}{16}$ & $9.998 \mathrm{e}-03$ & 1.13 & $8.033 \mathrm{e}-03$ & 1.25 & $8.044 \mathrm{e}-03$ & 1.25 \\
\hline$\frac{1}{32}$ & $4.672 \mathrm{e}-03$ & 1.10 & $3.473 \mathrm{e}-03$ & 1.21 & $3.481 \mathrm{e}-03$ & 1.21 \\
\hline$\frac{1}{64}$ & $2.236 \mathrm{e}-03$ & 1.06 & $1.568 \mathrm{e}-03$ & 1.14 & $1.573 \mathrm{e}-03$ & 1.15 \\
\hline$\frac{1}{128}$ & $1.090 \mathrm{e}-03$ & 1.04 & $7.364 \mathrm{e}-04$ & 1.09 & $7.393 \mathrm{e}-04$ & 1.09 \\
\hline
\end{tabular}

Table 4: $L^{2}$-norm error and convergence rate for Example 2.

\begin{tabular}{||c|c|c|c|c|c|c||}
\hline$h$ & $\left\|u_{h}-u\right\|$ & order & $\left\|u_{h}-u\right\|$ & order & $\left\|u_{h}-u\right\|$ & order \\
\hline \multicolumn{2}{|c|}{$\epsilon=1$} & \multicolumn{2}{|c|}{$\epsilon=10^{-3}$} & \multicolumn{2}{c||}{$\epsilon=10^{-9}$} \\
\hline$\frac{1}{4}$ & $5.822 \mathrm{e}-03$ & & $3.879 \mathrm{e}-03$ & & $3.869 \mathrm{e}-03$ & \\
\hline$\frac{1}{8}$ & $1.261 \mathrm{e}-03$ & 2.21 & $8.408 \mathrm{e}-04$ & 2.21 & $8.295 \mathrm{e}-04$ & 2.22 \\
\hline$\frac{1}{16}$ & $2.547 \mathrm{e}-04$ & 2.31 & $1.916 \mathrm{e}-04$ & 2.13 & $1.834 \mathrm{e}-04$ & 2.18 \\
\hline$\frac{1}{32}$ & $5.324 \mathrm{e}-05$ & 2.26 & $4.608 \mathrm{e}-05$ & 2.06 & $4.229 \mathrm{e}-05$ & 2.12 \\
\hline$\frac{1}{64}$ & $1.185 \mathrm{e}-05$ & 2.16 & $1.138 \mathrm{e}-05$ & 2.02 & $1.008 \mathrm{e}-05$ & 2.07 \\
\hline$\frac{1}{128}$ & $2.775 \mathrm{e}-06$ & 2.09 & $2.835 \mathrm{e}-06$ & 2.01 & $2.455 \mathrm{e}-06$ & 2.04 \\
\hline
\end{tabular}

\subsection{Example 3}

In this example, we consider the following singularly perturbed reaction-diffusion equations with variable coefficient:

$$
\begin{array}{ll}
-\epsilon \Delta u+(x+y) u=f & \text { in } \Omega, \\
u=0 & \text { on } \partial \Omega,
\end{array}
$$

where $0<\epsilon \leq 1$. The term $f$ is chosen according the exact solution $u=x y(1-x)(1-y)$. The optimal convergence rates for the corresponding MWG solutions are presented in 
Table 5: Discrete $H^{1}$-norm error and convergence rate for Example 3.

\begin{tabular}{||c|c|c|c|c|c|c||}
\hline$h$ & $\left\|e_{h}\right\|$ & order & $\left\|e_{h}\right\|$ & order & $\left\|e_{h}\right\|$ & order \\
\hline \multicolumn{2}{|c|}{$\epsilon=1$} & \multicolumn{2}{|c|}{$\epsilon=10^{-3}$} & \multicolumn{2}{c||}{$\epsilon=10^{-9}$} \\
\hline$\frac{1}{4}$ & $3.421 \mathrm{e}-02$ & & $3.066 \mathrm{e}-02$ & & $3.067 \mathrm{e}-02$ & \\
\hline$\frac{1}{8}$ & $1.531 \mathrm{e}-02$ & 1.16 & $1.262 \mathrm{e}-02$ & 1.28 & $1.263 \mathrm{e}-02$ & 1.28 \\
\hline$\frac{1}{16}$ & $7.103 \mathrm{e}-03$ & 1.11 & $5.355 \mathrm{e}-03$ & 1.24 & $5.366 \mathrm{e}-03$ & 1.23 \\
\hline$\frac{1}{32}$ & $3.368 \mathrm{e}-03$ & 1.07 & $2.370 \mathrm{e}-03$ & 1.18 & $2.378 \mathrm{e}-03$ & 1.17 \\
\hline$\frac{1}{64}$ & $1.628 \mathrm{e}-03$ & 1.05 & $1.095 \mathrm{e}-03$ & 1.11 & $1.099 \mathrm{e}-03$ & 1.11 \\
\hline$\frac{1}{128}$ & $7.984 \mathrm{e}-04$ & 1.03 & $5.222 \mathrm{e}-04$ & 1.07 & $5.246 \mathrm{e}-04$ & 1.07 \\
\hline
\end{tabular}

Table 6: $L^{2}$-norm error and convergence rate for Example 3.

\begin{tabular}{||c|c|c|c|c|c|c||}
\hline$h$ & $\left\|u_{h}-u\right\|$ & order & $\left\|u_{h}-u\right\|$ & order & $\left\|u_{h}-u\right\|$ & order \\
\hline \multicolumn{2}{|c|}{$\epsilon=1$} & \multicolumn{2}{c|}{$\epsilon=10^{-3}$} & \multicolumn{2}{c||}{$\epsilon=10^{-9}$} \\
\hline$\frac{1}{4}$ & $4.287 \mathrm{e}-03$ & & $2.602 \mathrm{e}-03$ & & $2.598 \mathrm{e}-03$ & \\
\hline$\frac{1}{8}$ & $8.718 \mathrm{e}-04$ & 2.30 & $5.674 \mathrm{e}-04$ & 2.20 & $5.607 \mathrm{e}-04$ & 2.21 \\
\hline$\frac{1}{16}$ & $1.746 \mathrm{e}-04$ & 2.32 & $1.319 \mathrm{e}-04$ & 2.10 & $1.274 \mathrm{e}-04$ & 2.14 \\
\hline$\frac{1}{32}$ & $3.711 \mathrm{e}-05$ & 2.23 & $3.207 \mathrm{e}-05$ & 2.04 & $3.009 \mathrm{e}-05$ & 2.08 \\
\hline$\frac{1}{64}$ & $8.414 \mathrm{e}-06$ & 2.14 & $7.950 \mathrm{e}-06$ & 2.01 & $7.290 \mathrm{e}-06$ & 2.05 \\
\hline$\frac{1}{128}$ & $1.995 \mathrm{e}-06$ & 2.08 & $1.983 \mathrm{e}-06$ & 2.00 & $1.793 \mathrm{e}-06$ & 2.02 \\
\hline
\end{tabular}

Tables 5 and 6 for $\epsilon=1, \epsilon=10^{-3}$ and $\epsilon=10^{-9}$. The numerical results demonstrate that the MWG solutions are convergent with order $\mathcal{O}(h)$ and $\mathcal{O}\left(h^{2}\right)$ in discrete $H^{1}$-norm and $L^{2}$-norm, respectively.

\subsection{Example 4}

In this example, we consider the following reaction-diffusion equations with variable coefficient:

$$
\begin{array}{cl}
-\nabla \cdot((x+y) \nabla u)+e^{x+y} u=f & \text { in } \Omega, \\
u=0 & \text { on } \partial \Omega .
\end{array}
$$

The term $f$ is chosen according the exact solution $u=\sin (\pi x) \sin (\pi y)$. The optimal convergence rates for the MWG solution are presented in Tables 7 and 8, which demonstrate that the MWG solutions are convergent with order $\mathcal{O}(h)$ and $\mathcal{O}\left(h^{2}\right)$ in discrete $H^{1}$-norm and $L^{2}$ norm, respectively.

All the numerical examples given above are in good agreement with the theoretical analysis in Section 6, which indicate that the MWG finite element method (3.8) is accurate and robust. 
Table 7: Discrete $H^{1}$-norm error and convergence rate for Example 4.

\begin{tabular}{||c|c|c||}
\hline$h$ & $\left\|e_{h}\right\|$ & order \\
\hline$\frac{1}{4}$ & $4.649 \mathrm{e}-01$ & \\
\hline$\frac{1}{8}$ & $2.196 \mathrm{e}-01$ & 1.08 \\
\hline$\frac{1}{16}$ & $1.058 \mathrm{e}-01$ & 1.05 \\
\hline$\frac{1}{32}$ & $5.141 \mathrm{e}-02$ & 1.04 \\
\hline$\frac{1}{64}$ & $2.524 \mathrm{e}-02$ & 1.03 \\
\hline$\frac{1}{128}$ & $1.249 \mathrm{e}-02$ & 1.01 \\
\hline
\end{tabular}

Table 8: $L^{2}$-norm error and convergence rate for Example 4.

\begin{tabular}{||c|c|c||}
\hline$h$ & $\left\|u_{h}-u\right\|$ & order \\
\hline$\frac{1}{4}$ & $5.409 \mathrm{e}-02$ & \\
\hline$\frac{1}{8}$ & $1.102 \mathrm{e}-02$ & 2.30 \\
\hline$\frac{1}{16}$ & $2.327 \mathrm{e}-03$ & 2.24 \\
\hline$\frac{1}{32}$ & $5.214 \mathrm{e}-04$ & 2.15 \\
\hline$\frac{1}{64}$ & $1.224 \mathrm{e}-04$ & 2.09 \\
\hline$\frac{1}{128}$ & $2.960 \mathrm{e}-05$ & 2.05 \\
\hline
\end{tabular}

\section{Conclusions}

We have presented a robust modified weak Galerkin finite element scheme for reaction-diffusion equations. The method eliminates the unknowns associated with the element boundaries in the WG method by introducing the average and the jump. As a result, the MWG method has fewer degrees of freedom than that of the WG method. In the MWG method, the finite element space and test functions space are the same space and the error $e_{h}=u_{h}-Q_{0} u$ belongs to the test functions space. This has contributed to the errors analysis for reaction-diffusion equations. We have established optimal-order error for the numerical approximation in $H^{1}$-norm and $L^{2}$-norm. The numerical experiments indicate that the MWG scheme is accurate and robust even when the solved equations are the singularly perturbed reaction-diffusion equations.

\section{Acknowledgments}

This work is supported by the State Key Program of National Natural Science Foundation of China (Grant 11931003), the National Natural Science Foundation of China (Grants 41974133, 11971410) and the Natural Science Foundation of Lingnan Normal University (Grant ZL2038).

\section{References}

[1] M. AinsworTh, I. BABUŠKA, Reliable and robust a posteriori error estimation for singularly perturbed reaction-diffusion problems, SIAM J. Numer. Anal. 36 (1999), 331-353.

[2] G. CHEn, M. FEng, X. XIE, A robust WG finite element method for convection-diffusionreaction equations, J. Comput. Appl. Math. 315 (2017), 107-125.

[3] L. Chen, J. WAng, Y. WAng, X. YE, An auxiliary space multigrid preconditioner for the weak Galerkin method, Comput. Math. Appl. 70 (2015), 330-344.

[4] L. Chen, J. WANG, X. YE, A posterior error estimates for weak Galerkin finite element methods for second-order elliptic problems, J. Sci. Comput. 59 (2014), 496-511.

[5] F. GAO, X. WANG, L. MU, A modified weak Galerkin finite element methods for convectiondiffusion problems in 2D, J. Appl. Math. Comput. 49 (2015), 493-511. 
[6] Y. HAN, H. LI, X. XIE, Robust globally divergence-free weak Galerkin finite element methods for unsteady natural convection problems, Numer. Math. Theor. Meth. Appl. 12 (2019), 1266-1308.

[7] G. KUNERT, Robust a posteriori error estimation for a singularly perturbed reaction-diffusion equation on anisotropic tetrahedral meshes, Adv. Comput. Math. 15 (2001), 237-259.

[8] B. LI, X. XIE, A two-level algorithm for the weak Galerkin discretization of diffusion problems, J. Comput. Appl. Math. 287 (2015), 179-195.

[9] G. Li, Y. ChEn, Y. HuAng, A new weak Galerkin finite element scheme for general secondorder elliptic problems, J. Comput. Appl. Math. 344 (2018), 701-715.

[10] J. LI, Uniform convergence of discontinuous finite element methods for singularly perturbed reaction-diffusion problems, Comput. Math. Appl. 44 (2002), 231-240.

[11] G. Lin, J. LIU, F. SADRE-MARANDI, A comparative study on the weak Galerkin, discontinous Galerkin, and mixed finite element methods, J. Comput. Appl. Math. 273 (2015), 346-362.

[12] R. Lin, Discontinuous discretization for least-squares formulation of singularly perturbed reaction-diffusion problems in one and two dimensions, SIAM J. Numer. Anal. 47 (2008), 89-108.

[13] R. Lin, Discontinuous Galerkin least-squares finite element methods for singularly perturbed reaction-diffusion problems with discontinuous coefficients and boundary singularities, $\mathrm{Nu}$ mer. Math. 112 (2009), 295-318.

[14] J. LiU, S. TAVENER, Z. WANG, Lowest-order weak Galerkin finite element method for Darcy flow on convex polygonal meshes, SIAM J. Sci. Comput. 40 (2018), 1229-1252.

[15] X. LIU, J. LI, Z. CHEN, A weak Galerkin finite element method for the Oseen equations, Adv. Comput. Math. 42 (2016), 1473-1490.

[16] L. MU, J. WANG, Y. WANG, X. YE, A computational study of the weak Galerkin method for second-order elliptic equations, Numer. Algor. 63 (2012), 753-777.

[17] L. Mu, J. WANG, G. WEI, X. YE, S. ZHAO, Weak Galerkin finite element methods for the elliptic interface problem, J. Comput. Phy. 250 (2013), 106-125.

[18] L. MU, J. WANG, X. YE, Weak Galerkin finite element methods for the biharmonic equation on polytopal meshes, Numer. Meth. Part. D E 30 (2014), 1003-1029.

[19] L. MU, J. WANG, X. YE, A weak Galerkin finite element method with polynomial reduction, J. Comput. Appl. Math. 285 (2015), 45-48.

[20] L. Mu, J. WANG, X. YE, A new weak Galerkin finite element method for the Helmholtz equation, IMA J. Numer. Anal. 35 (2015), 1228-1255.

[21] L. MU, J. WANG, X. YE, AND S. ZHANG, A $C^{0}$-weak Galerkin finite element method for the biharmonic equation, J. Sci. Comput. 59 (2014), 473-495.

[22] L. MU, J. WANG, X. YE, AND S. ZHANG, A weak Galerkin finite element method for the Maxwell equations, J. Sci. Comput. 65 (2015), 363-386.

[23] L. MU, J. WANG, X. YE, S. ZHAO, A numerical study on the weak Galerkin method for the Helmholtz equation, Commun. Comput. Phys. 15 (2014), 1461-1479.

[24] L. MU, J. WANG, X. YE, S. ZHAO, A new weak Galerkin finite element method for elliptic interface problems, J. Comput. Phy. 325 (2016), 157-173.

[25] L. MU, X. WANG, X. YE, A modified weak Galerkin finite element method for the Stokes equations, J. Comput. Appl. Math. 275 (2015), 79-90.

[26] H. PENG, X. WANG, Q. ZHAI AND R. ZHANG, A weak Galerkin finite element method for the elliptic variational inequality, Numer. Math. Theor. Meth. Appl. 12 (2019), 923-941.

[27] C. WANG AND J. WANG, An efficient numerical scheme for the biharmonic equation by weak Galerkin finite element methods on polygonal or polyhedral meshes, Comput. Math. Appl. 68 (2014), 2314-2330. 
[28] J. WANG, X. YE, A weak Galerkin finite element method for second-order elliptic problems, J. Comput. Appl. Math. 241 (2013), 103-115.

[29] J. WANG, X. YE, A weak Galerkin mixed finite element method for second-order elliptic problems, Math. Comp. 83 (2014), 2101-2126.

[30] J. WANG, X. YE, A weak Galerkin finite element method for the Stokes equations, Adv. Comput. Math. 42 (2016), 155-174.

[31] X. Wang, N. S. Malluwawadu, F. GaO, T. C. McMillan, A modified weak Galerkin finite element method, J. Comput. Appl. Math. 271 (2014), 319-327.

[32] T. P. WiHLER, B.RIVIERE, Discontinuous Galerkin methods for second-order elliptic PDE with low-regularity solutions, J. Sci. Comput. 46 (2011), 151-165.

[33] Q. ZHAI, R. ZHANG, X. WANG, A hybridized weak Galerkin finite element scheme for the Stokes equations, Sci. China Math. 58 (2015), 2455-2472.

[34] Q. ZHANG, H. KUANG, X. WANG AND Q. ZHAI, A hybridized weak Galerkin finite element method for incompressible Stokes equations, Numer. Math. Theor. Meth. Appl. 12 (2019), 1012-1038.

[35] R. ZHANG AND Q. ZHAI, A weak Galerkin finite element scheme for the biharmonic equations by using polynomials of reduced order, J. Sci. Comput. 64 (2015), 559-585.

[36] J. ZHou, D. Xu, H. Chen, A weak Galerkin finite element method for multi-term timefractional diffusion equations, East Asian J. Appl. Math. 8 (2018) 181-193.

[37] J. ZHOU, D. XU, X. DAI, Weak Galerkin finite element method for the parabolic integrodifferential equation with weakly singular kernel, Comput. Appl. Math. 38 (2019), 38. 